\title{
Multivalent, Soluble Nano-Self Peptides Increase Phagocytosis of Antibody-Opsonized Targets while Suppressing 'Self' Signaling
}

AbdelAziz R. Jalil, Brandon H. Hayes, Jason C. Andrechak, Yuntao Xia, David M. Chenoweth, Dennis E. Discher

\section{Supplementary Methods}

\section{SIRPa expression and purification}

Soluble human SIRPa fused with glutathione s-transferase (SIRPa-GST) was expressed and purified as previously published. ${ }^{1}$

\section{nano-Self peptides binding to soluble SIRPa}

a- Peptide biotinylation

NHS-biotin (Thermo Fisher) was mixed with nS-wt and nS-V, respectively, following the manufacturer's protocol. The biotinylated peptides were dialyzed against PBS.

b- Streptavidin-beads binding

Streptavidin-coated polystyrene beads of $2.1 \mu \mathrm{m}$ radius (Spherotech) were washed and blocked three times in PBS plus $0.4 \% \mathrm{w} / \mathrm{v}$ bovine serum albumin (BSA; Sigma). nS-wtbiotin or nS-V-biotin were incubated with the beads for 1 hour at room temperature with shaking. Beads were washed with PBS, then incubated with recombinant SIRPa for 1 hour on ice. Streptavidin beads were labeled with rabbit anti-streptavidin-FITC (Invitrogen), and SIRPa was stained with mouse anti-SIRPa-allophycocyanin (anti-SIRPa-APC; Invitrogen).

\section{Affinity binding assay}

The same binding protocol mentioned above was used. Increasing concentrations (10 nM to 1 $\mathrm{mM}$ ) of FAM-labeled peptides were incubated with THP-1 macrophages before or after fixation. For J774A.1 macrophages, affinity binding assay was only done on live cells. Fluorescence imaging and analysis were done as described in Methods.

\section{Opsonin Titration}

The same phagocytosis protocol as described in Methods was used. Increasing concentrations (from $33 \mathrm{nM}$ to $1.33 \mu \mathrm{M}$ ) of anti-RBC lgG were incubated with the RBCs. CD47 on RBCs was blocked in all conditions with $5 \mu \mathrm{g} / \mathrm{mL}$ anti-CD47. Fluorescence imaging and analysis were done as described in Methods. 


\section{Supplementary Figures}

Fig. S1: Analytical HPLC traces and MALDI-TOF mass confirmation of all peptides indicate successful and pure synthesis

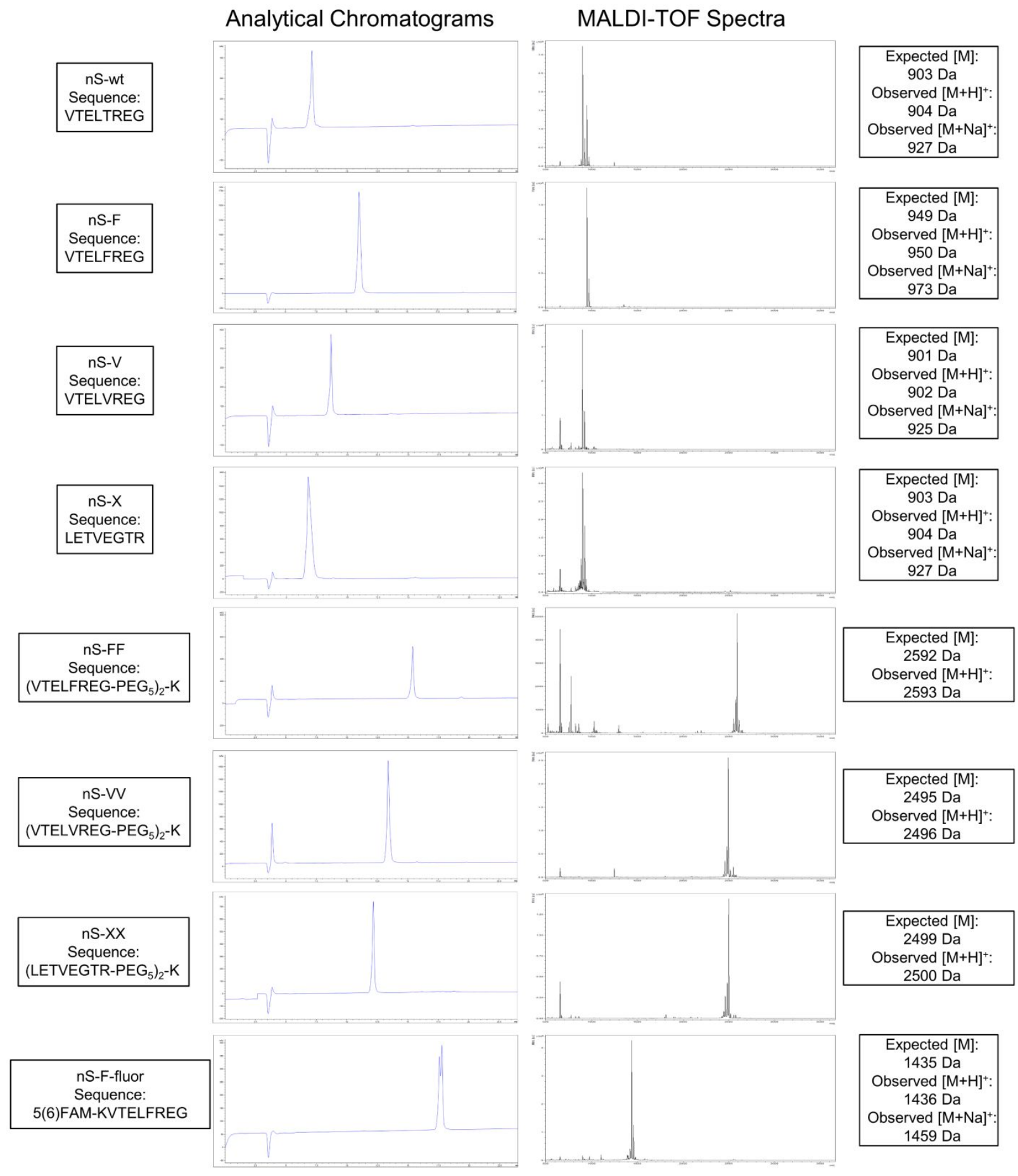


Figure S1: Synthesis and purity of nano-self peptides are characterized and confirmed by analytical HPLC and MALDI-TOF mass spectrometry

All peptides used in this assay were run on an analytical HPLC to determine purity of the nS peptides. All analytical traces show one major peak for each peptide. The peptides were all characterized by MALDI-TOF mass spectrometry. Single main peaks appear for the correct mass for all peptides. 
Fig. S2: PMA differentiated THP-1 macrophages have an identical gene profile as primary mouse macrophages based on microarray gene expression analysis

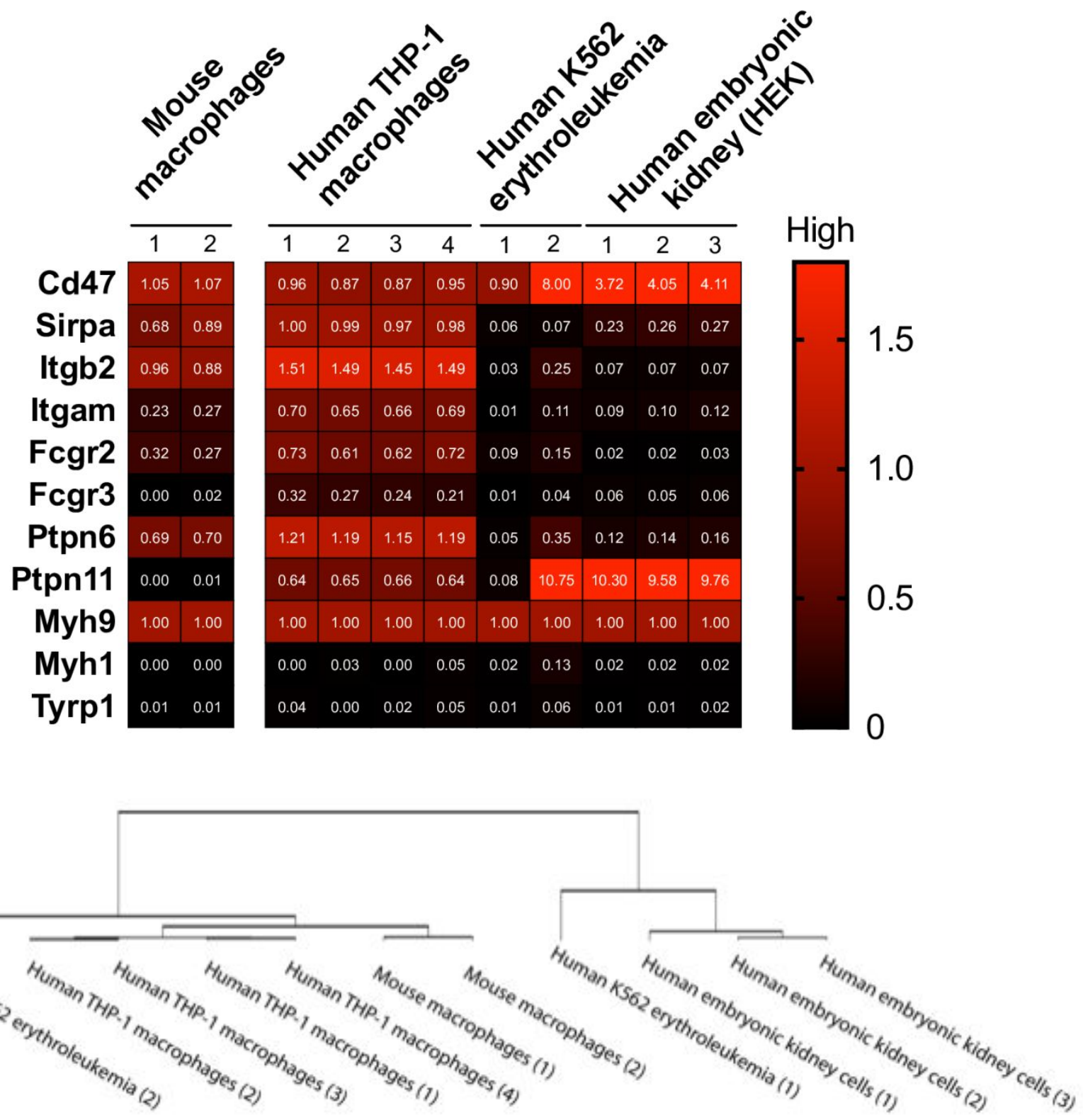


Figure S2: PMA differentiated THP-1 cells share identical gene profiles as primary macrophages for key, pathway-relevant factors.

(Top) Microarray gene expression analysis ${ }^{2}$ verification that PMA differentiated THP-1 macrophages express several key macrophage factors at similar levels as primary mouse macrophages (i.e. Sirpa, the Integrin (Itg) genes, Fcgr genes, and SHP1 gene Ptpn6), while differing from other hematopoietic and non-hematpoietic cells (K562 and HEK, respectively). Positive control genes are the ubiquitous Cd47 and the widely expressed nonmuscle myosin-II gene, Myh9. Negative control genes are skeletal muscle myosin, Myh1, and melanin synthesis gene, Tyrp1.

(Bottom) Dendrogram from hierarchical clustering analysis validating similar profiles between marrow-derived mouse macrophages and PMA-differentiated THP-1 macrophages while clearly distinct from other cell types. 
Fig. S3:

A. Sequence of tetravalent nano-Self peptide "nS-F4"

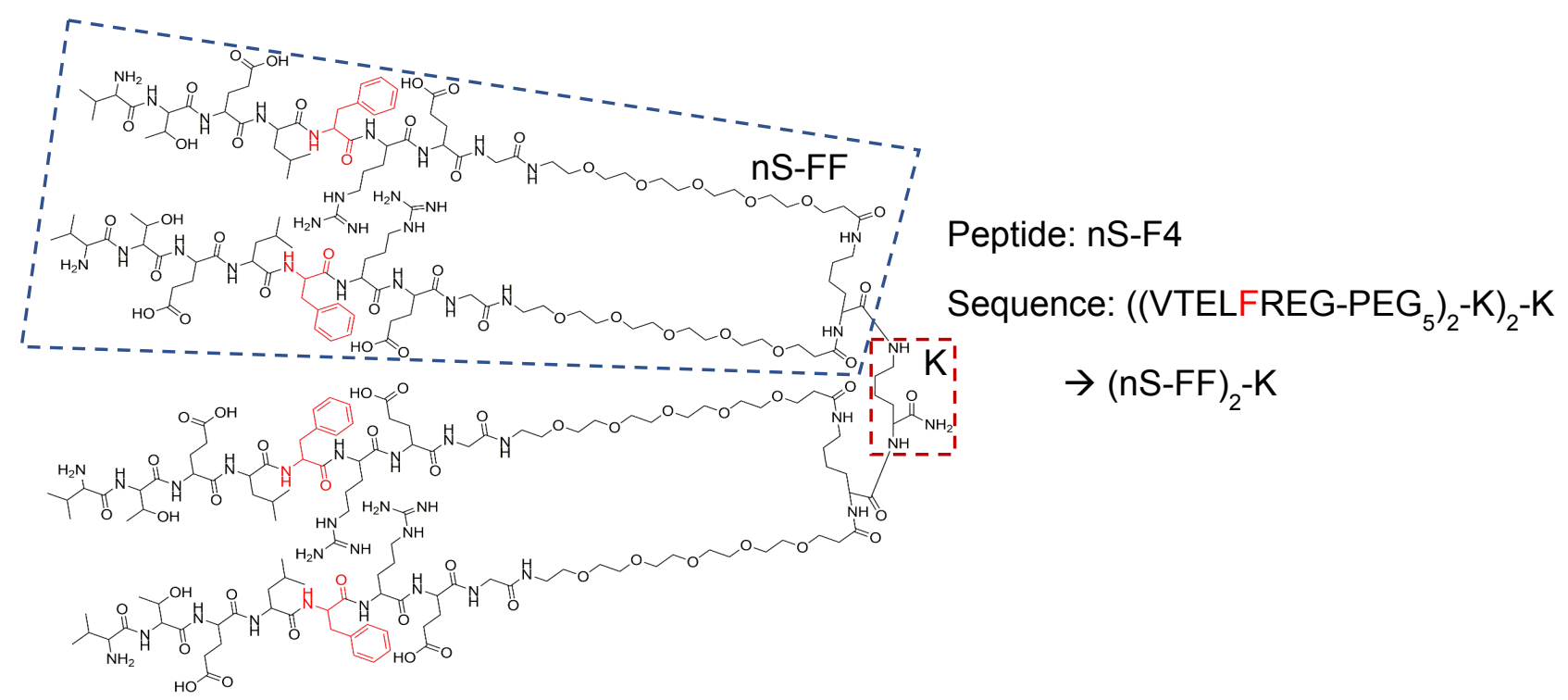

B. Analytical HPLC chromatogram and MALDI-TOF mass spectrum indicate successful synthesis of tetravalent nS-F4

HPLC: $5-40 \% \mathrm{CH}_{3} \mathrm{CN}$ in $0.1 \% \mathrm{TFA} / \mathrm{H}_{2} \mathrm{O}$ over 24 minutes at room temperature

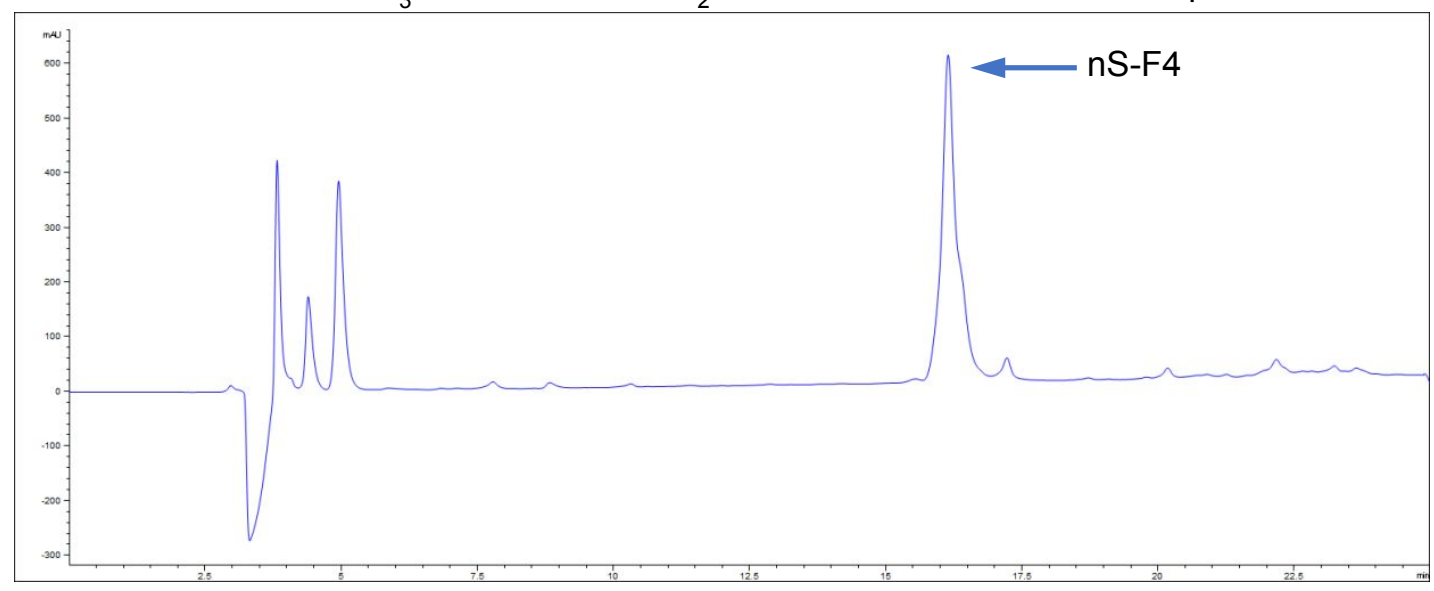

MALDI-TOF: $2 \mu \mathrm{L}$ CHCA matrix $+2 \mu \mathrm{L}$ of sample

Expected mass [M]: 5295 Da Observed mass [M] ${ }^{+}: 5295 \mathrm{Da}$

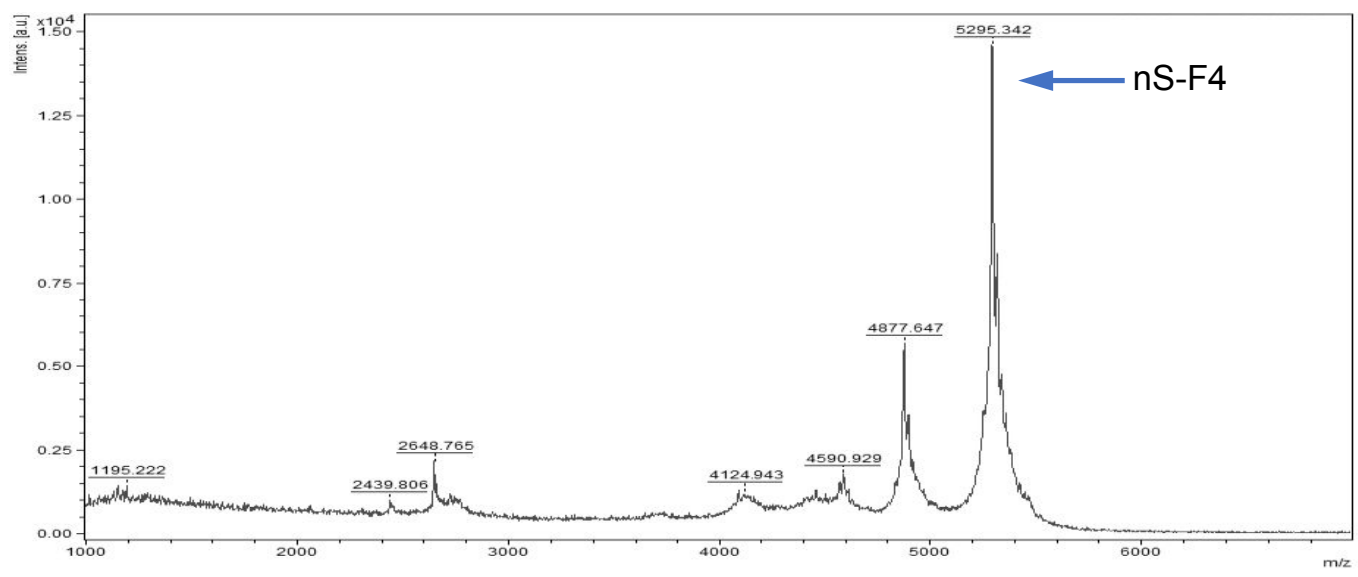


Figure S3: Successful synthesis and purification of tetravalent nano-Self-F peptide (nS-F4)

A. Chemical structure sketch of tetravalent nano-Self peptide.

B. One main peak was observed in the analytical trace and MALDI-TOF chromatogram corresponding to the correct mass of nS-F4 indicating successful and pure synthesis of nS-F4. 
Fig. S4: Phagocytosis levels are significantly greater than all control conditions when macrophages are incubated with bivalent nano-Self peptides

\begin{tabular}{|c|c|c|c|c|c|c|c|c|c|c|}
\hline & $\begin{array}{l}+50 \mu \mathrm{MX} \\
+ \text { anti-RBC } \\
\end{array}$ & $\begin{array}{l}+50 \mu \mathrm{M} X \mathrm{XX} \\
+ \text { anti-RBC } \\
\end{array}$ & \begin{tabular}{|l|}
$+50 \mu \mathrm{M} w \mathrm{t}$ \\
+ anti-RBC \\
\end{tabular} & $\begin{array}{r}+50 \mu \mathrm{M} \mathrm{V} \\
+ \text { anti-RBC } \\
\end{array}$ & $\begin{array}{l}+50 \mu \mathrm{M} \mathrm{VV} \\
+ \text { anti-RBC }\end{array}$ & $\begin{array}{r}+50 \mu \mathrm{M} \mathrm{F} \\
+ \text { anti-RBC } \\
\end{array}$ & $\begin{array}{l}+50 \mu \mathrm{M} \mathrm{FF} \\
+ \text { anti-RBC } \\
\end{array}$ & $\begin{array}{l}+ \text { anti-CD47 } \\
+ \text { anti-RBC }\end{array}$ & + anti-RBC & + anti-CD47 \\
\hline RBCs alone & * & * & * & * & * & * & * & * & * & * \\
\hline + anti-CD47 & * & & * & * & * & * & * & * & * & \\
\hline+ anti-RBC & & & * & * & * & * & * & * & & \\
\hline $\begin{array}{l}+ \text { anti-CD47 } \\
+ \text { anti-RBC }\end{array}$ & & * & & * & * & * & * & & & \\
\hline $\begin{array}{l}+50 \mu \mathrm{M} \mathrm{FF} \\
+ \text { anti-RBC }\end{array}$ & * & * & * & & & & & & & \\
\hline \begin{tabular}{|l|}
$+50 \mu \mathrm{M} \mathrm{F}$ \\
+ anti-RBC \\
\end{tabular} & * & * & * & & & & & & & \\
\hline $\begin{array}{l}+50 \mu \mathrm{M} \mathrm{VV} \\
+ \text { anti-RBC }\end{array}$ & * & * & * & & & & & & & \\
\hline $\begin{array}{l}+50 \mu \mathrm{M} \mathrm{V} \\
+ \text { anti-RBC }\end{array}$ & * & * & & & & & & & & \\
\hline $\begin{array}{l}+50 \mu \mathrm{M} w \mathrm{t} \\
+ \text { anti-RBC }\end{array}$ & & * & & & & & & & & \\
\hline $\begin{array}{l}+50 \mu \mathrm{M} X \mathrm{XX} \\
+ \text { anti-RBC }\end{array}$ & & & & & & & & & & \\
\hline
\end{tabular}

Figure S4: Treatment of human macrophages with multivalent nano-Self peptides enhances phagocytosis levels significantly relative to peptide and antibody controls All conditions were compared with each other to determine which conditions were significantly different ( ${ }^{*}$ denotes $p<0.05$ for statistical significance between conditions). Both multivalent peptides enhanced macrophage phagocytosis significantly compared to all control conditions. 
Fig. S5: Anti-RBC titration curves to determine amount of opsonin needed for optimal and comparable engulfment of target cells by macrophages
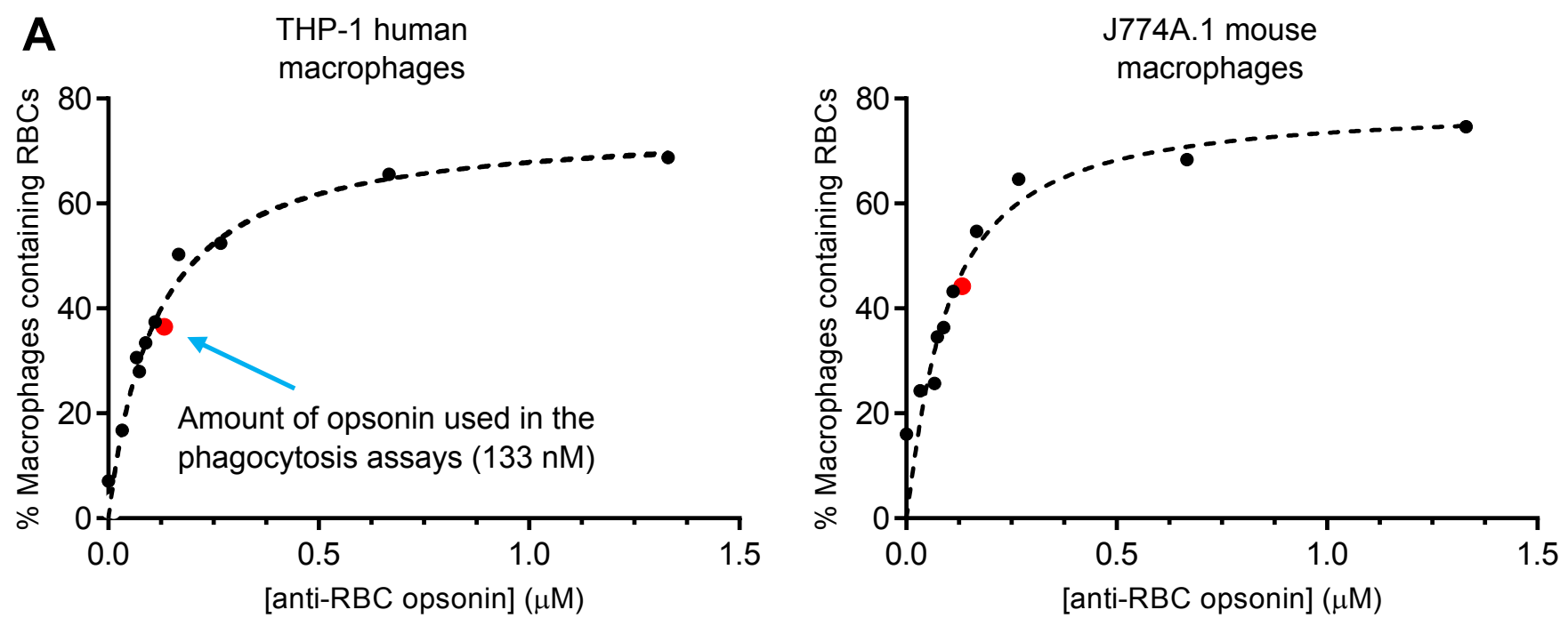

B

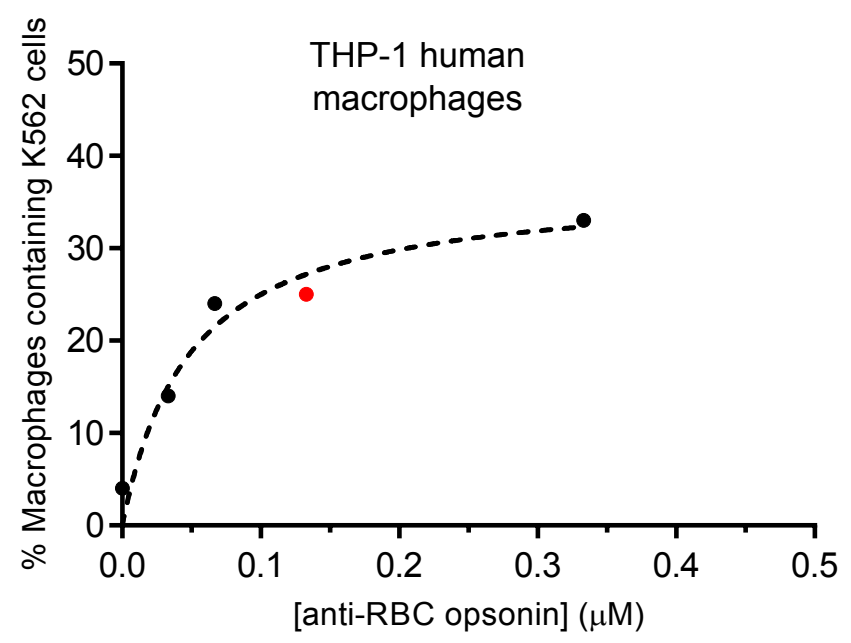

Soluble nano-Self peptides enhance phagocytosis of opsonized red blood cells in J774A.1 mouse macrophages

C

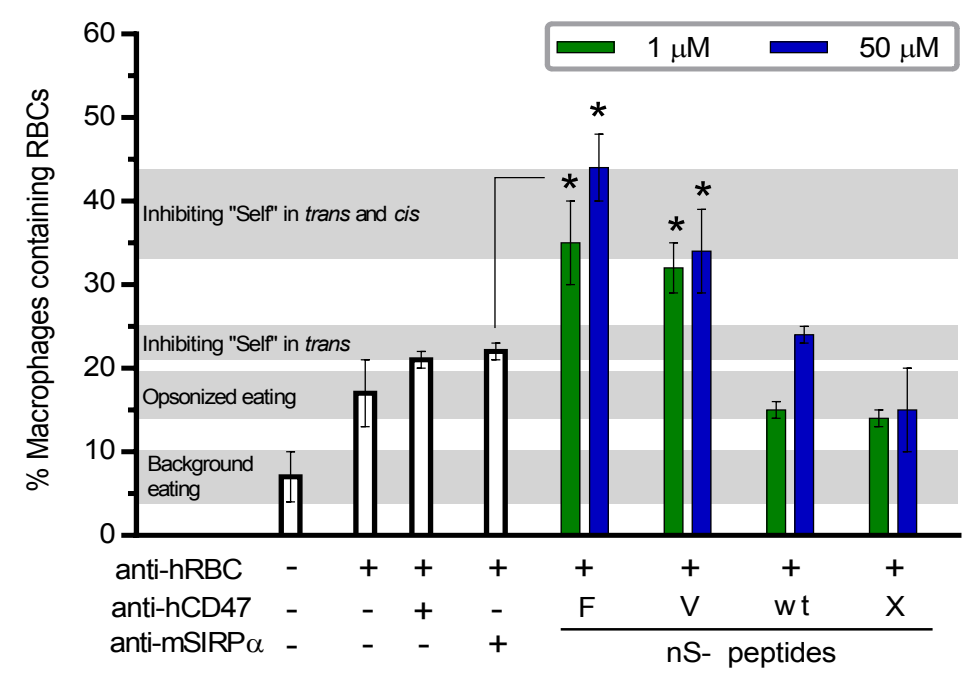


Figure S5: Using optimal concentration of opsonin allows for comparison of macrophage phagocytosis

Varying concentrations of anti-RBC opsonin incubated with CD47-blocked RBCs (A) and K562 cells (B) to determine the optimal amount of opsonization necessary for optimal phagocytosis. Human and mouse macrophages responded differently to RBC opsonization, which may be a result of the polyclonal antibody and how it engages with FcRs on the respective macrophages. The optimal concentration for phagocytosis assays was selected to be $133 \mathrm{nM}$ (red point) because it gave a reasonable phagocytosis response far from baseline and saturation.

C. Treating mouse macrophages with nS peptides results in enhanced engulfment of opsonized RBCs. 
Fig. S6: Greater number of CD47 molecules are on the surface of human erythroleukemia K562 cells when compared to healthy red blood cells

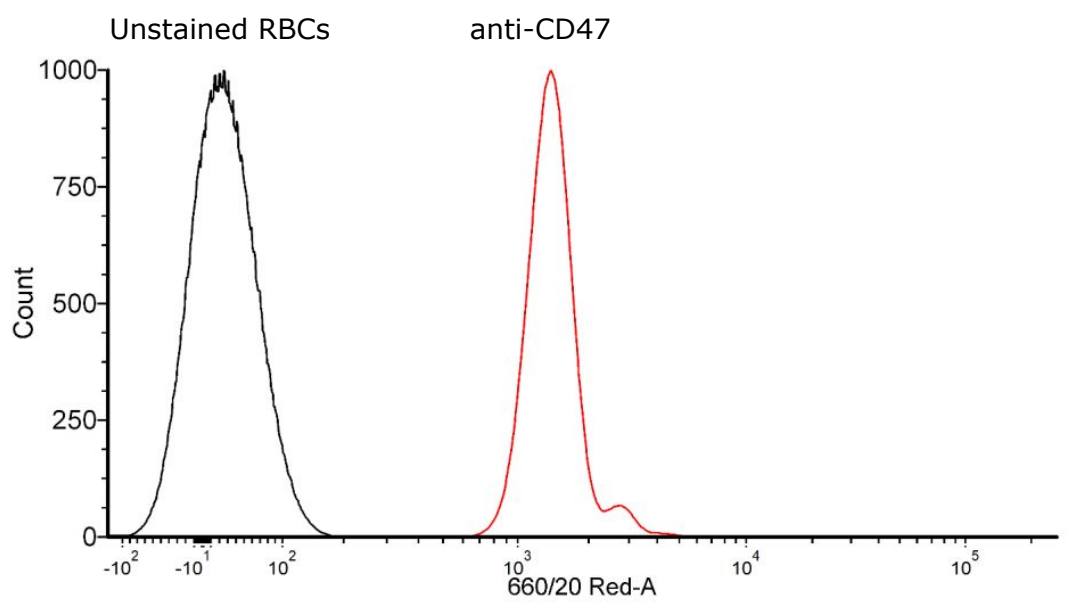

$$
\text { Unstained } \mathrm{K} 562 \text { cells anti-CD47 }
$$

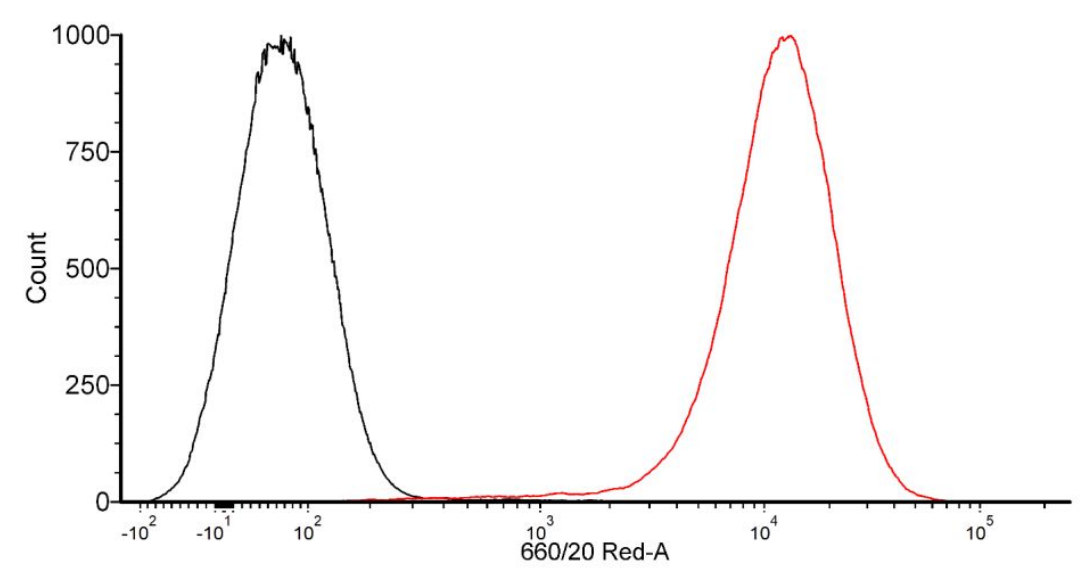

Figure S6: CD47 expression on RBCs and K562 cancer cells

Flow cytometry quantitation of primary fluorescent anti-CD47 antibody. Samples were analyzed the same day using the same voltage settings on the flow cytometer. Higher numbers of CD47 molecules are present on the surface of K562 cells when compared to RBCs. 
Fig. S7: CD47-Fc binding assay to determine apparent binding constant for THP-1

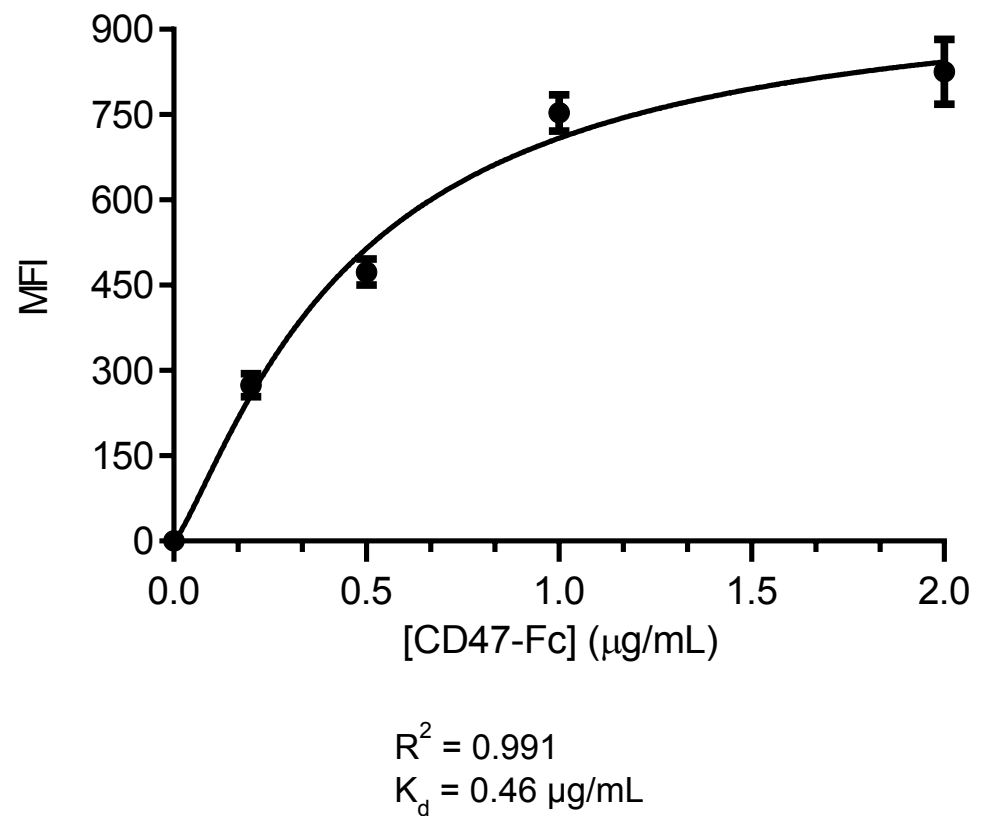

Figure S7: CD47-Fc binding curve

Varying concentrations of CD47-Fc incubated with human THP-1 macrophages and measured by anti-Fc fluorescence. Apparent $K_{d}$ was determined to be $0.46 \mu \mathrm{g} / \mathrm{mL}$. 
Fig. S8: Bivalent nano-Self peptides outcompete monovalent nano-Self in interacting with macrophages
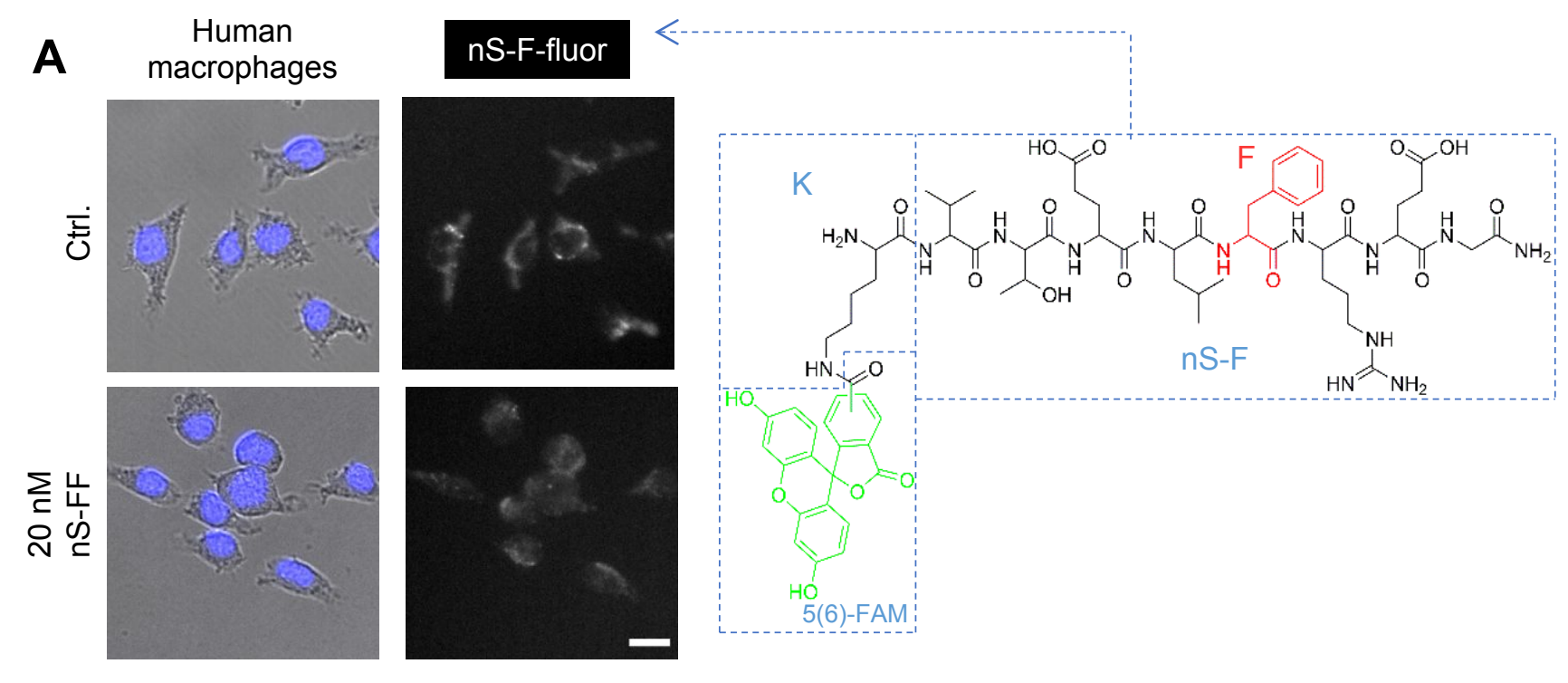

B

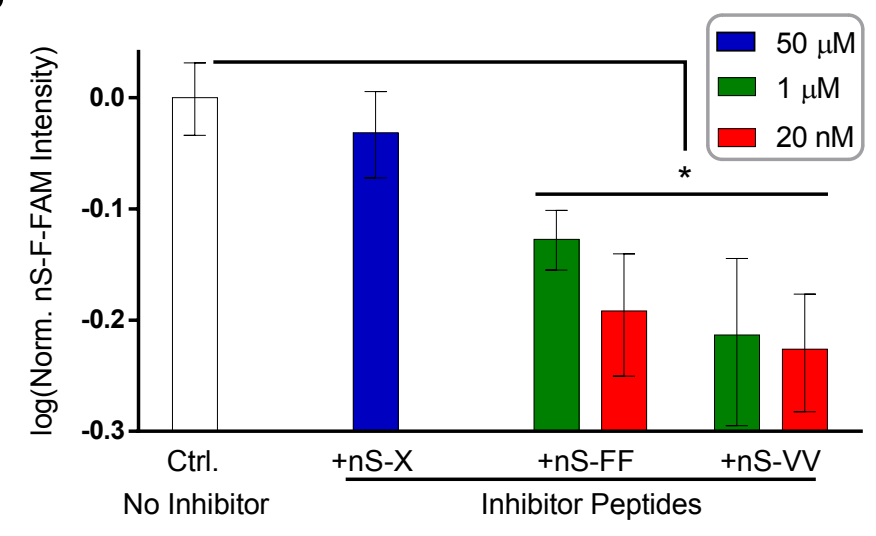

Figure S8: Multivalency enhances peptide affinity for macrophages

A. Representative fluorescence microscopy images of nS-F-fluor fluorescence inhibition on macrophages after addition of multivalent nS peptide inhibitors (scale bar: $25 \mu \mathrm{m}$ ).

B. Bivalent peptides show higher affinity towards macrophages by outcompeting the binding of

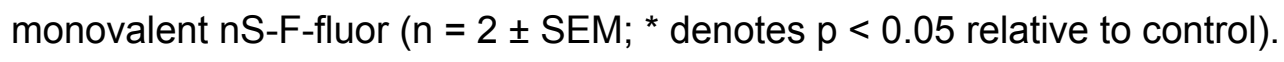


Fig. S9: Immobilized nano-Self peptides bind to recombinant SIRPa

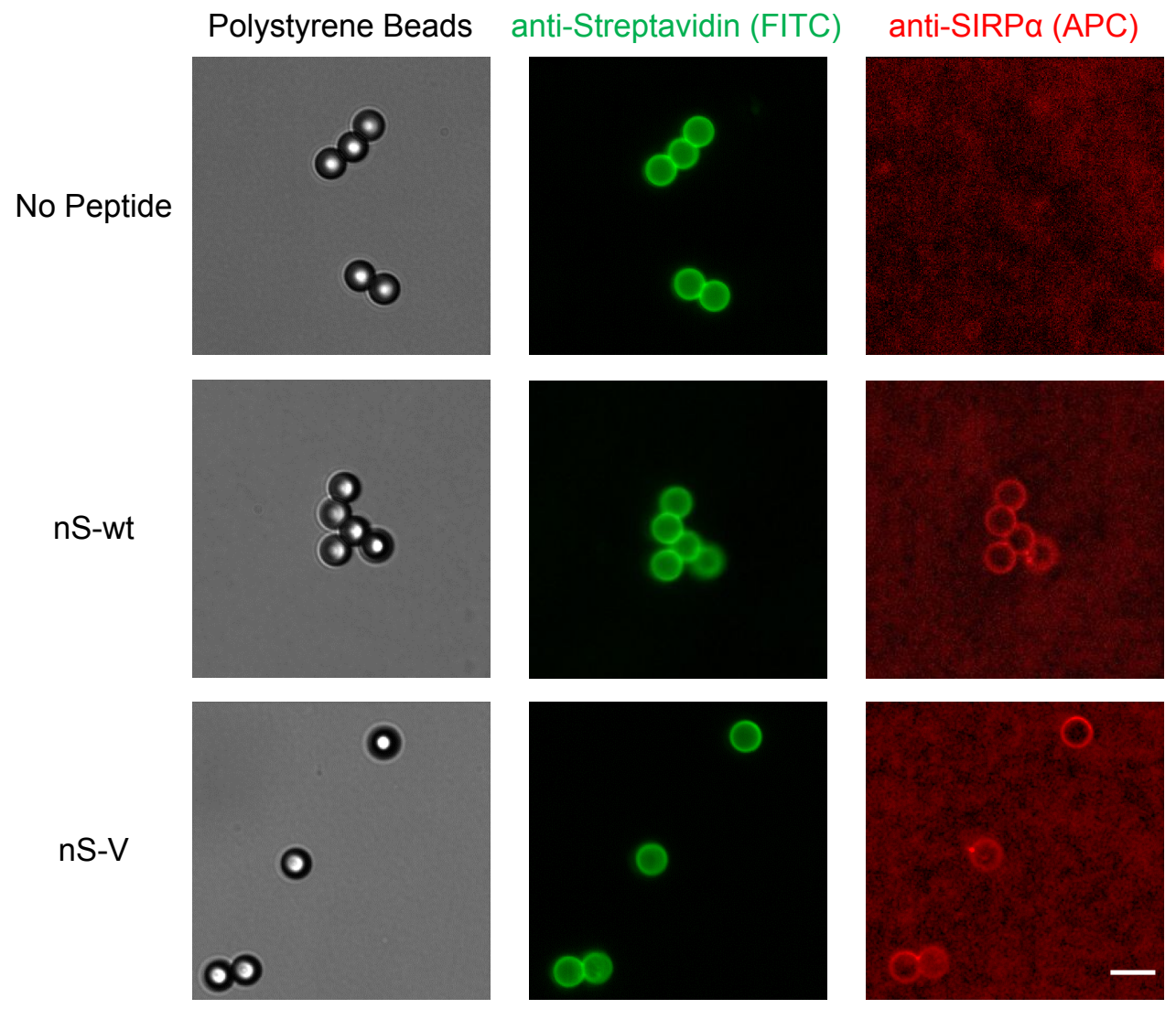

Figure S9: Immobilized nano-Self peptides bind to soluble SIRPa

Polystyrene beads coated with streptavidin incubated with or without biotinylated nS-wt or nS-V. After immobilizing the nS peptides, recombinant CD47-binding domain of SIRPa was added. SIRPa was then stained with nonblocking APC-labeled primary antibody. Fluorescence is only observed in the presence of biotinylated peptide (scale bar: $5 \mu \mathrm{m}$ ). 
Fig. 10: nano-Self peptides bind SIRPa on macrophages

A

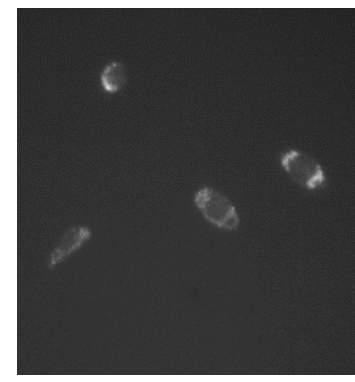

nS-V-fluor

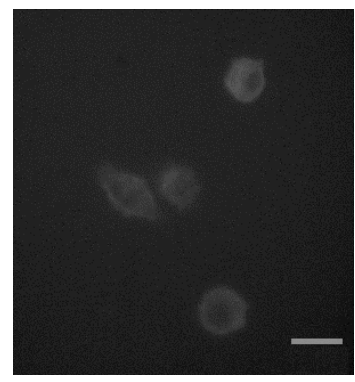

P84-FITC

B
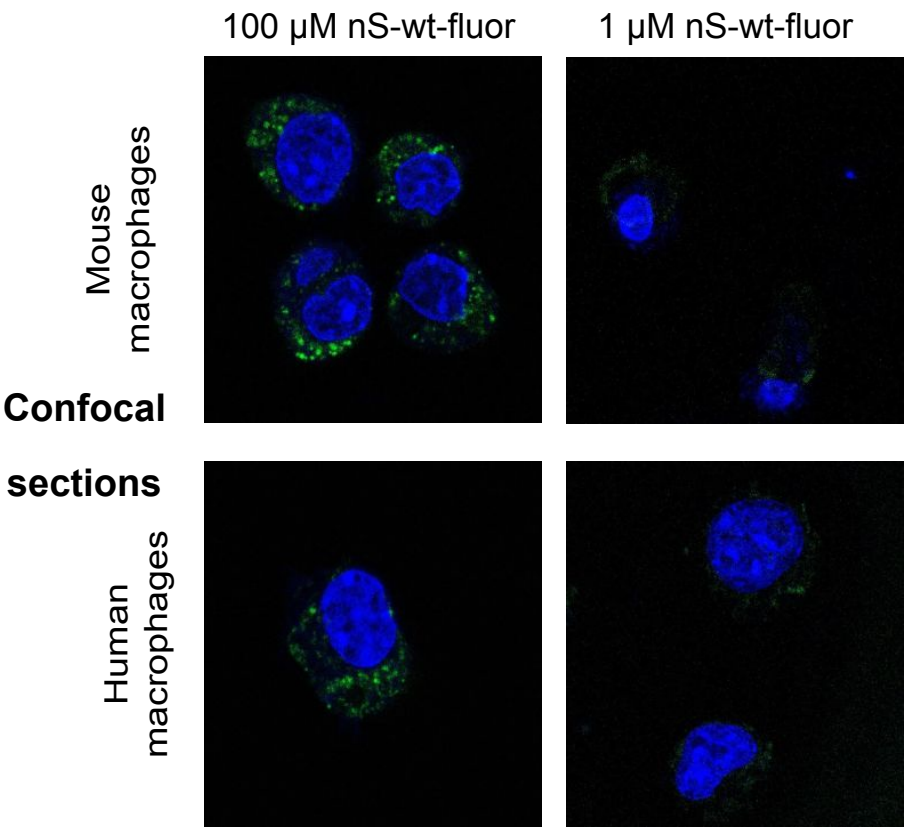

$1 \mu \mathrm{M}$ nS-X-fluor
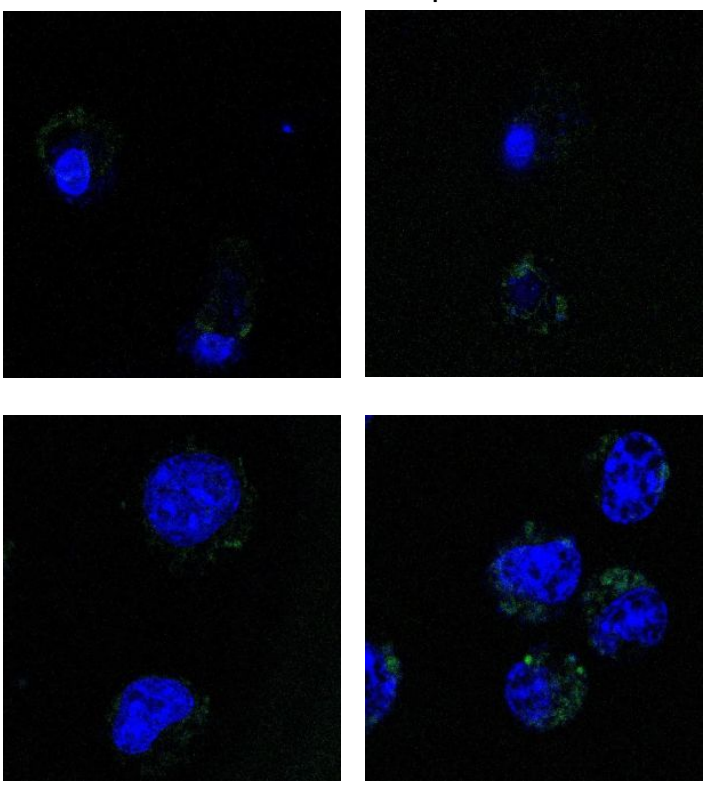

C

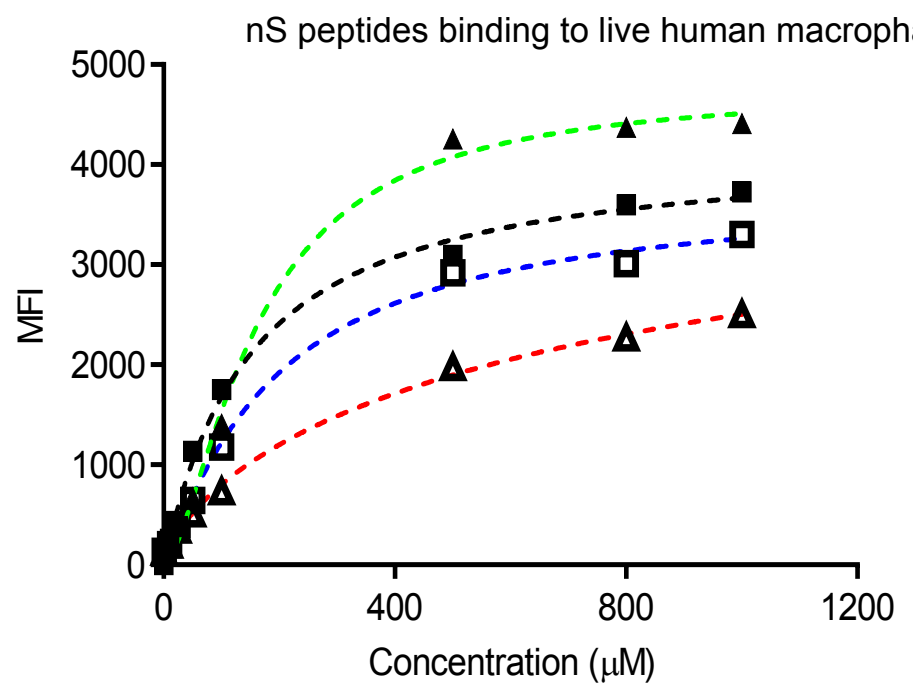

- - nS-wt-fluor

- $\boldsymbol{\Delta}$ - nS-V-fluor

-E. nS-F-fluor

-A. nS-X-fluor 
Figure S10: nano-Self peptides bind to membrane bound SIRPa and are also internalized by human and mouse macrophages

A. Fluorescence images of $\mathrm{nS}-\mathrm{V}$-fluor and P84-FITC bound to the surface of live mouse macrophages (scale bar $25 \mu \mathrm{m}$ ).

B. Confocal z-stack images of mouse and human macrophages incubated with FAM-labelled peptides. In addition to membrane binding, internalization of nS-wt occurs more readily at higher concentrations leading to higher fluorescence intensity. At low concentrations ( $1 \mu \mathrm{M})$, the peptide is mainly internalized with minimal membrane staining hence the decrease in fluorescence.

C. Fluorescent $\mathrm{nS}$ peptides binding to the surface of live human macrophages. 
Fig. S11: Monovalent nano-Self peptides also exhibit some hairpin like structure
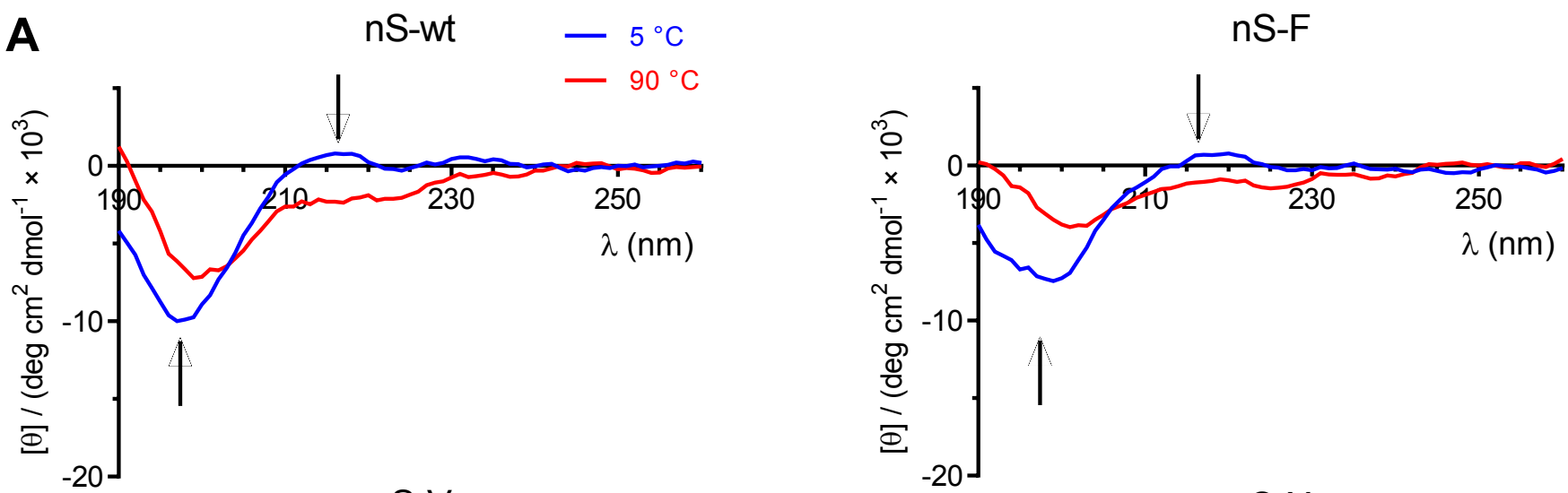

$\mathrm{nS}-\mathrm{V}$
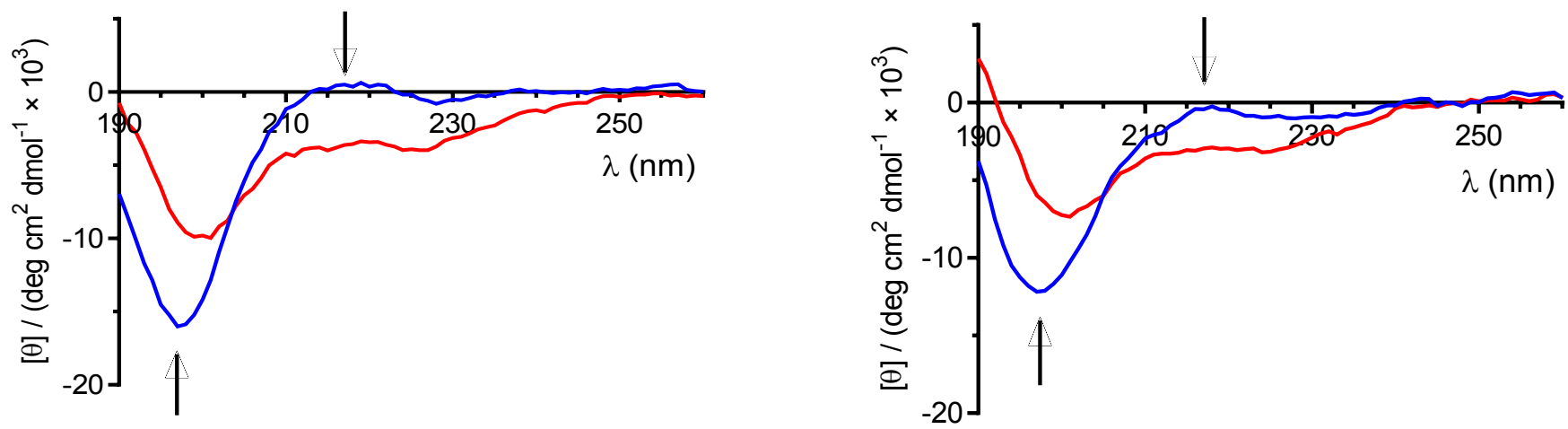

B Random coil/ß-turn

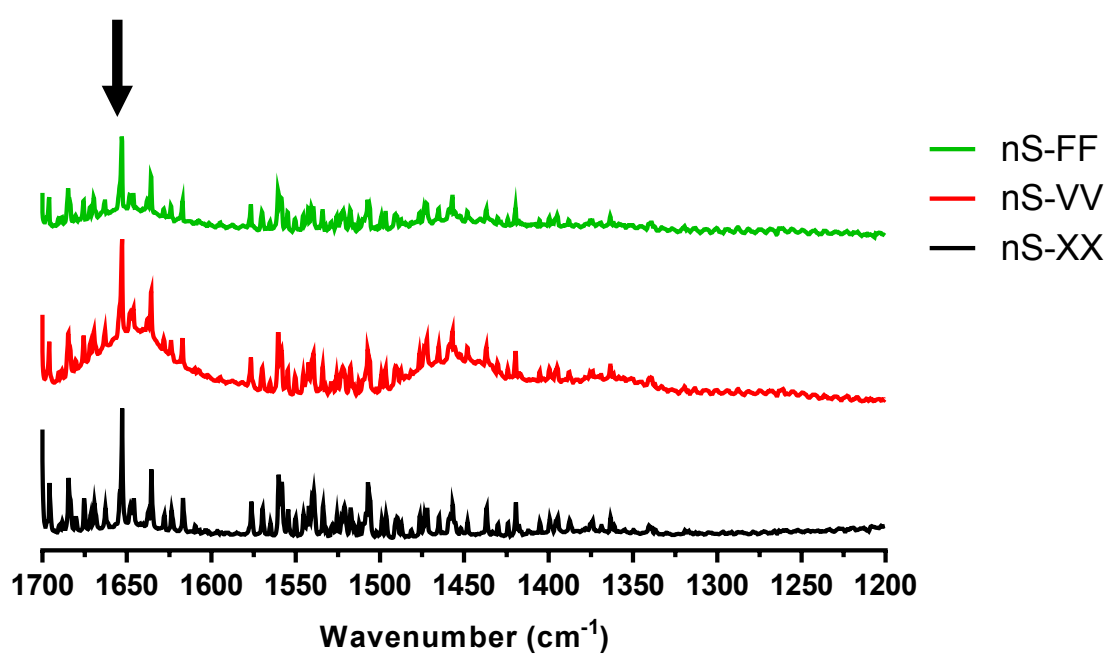

Figure S11: nano-Self peptides conform to some $\beta$-hairpin structure but mainly random coil

A. CD spectra of monovalent $n S$ peptides.

B. FT-IR spectra of bivalent nS peptides. Main peak appears near $1650 \mathrm{~cm}^{-1}$, which is a characteristic peak shared by both random coil and $\beta$-turn peptides. 
Fig. S12: Tetravalent nS-F4 inhibits bivalent association with macrophages and apparently has stronger avidity for SIRPa than bivalent

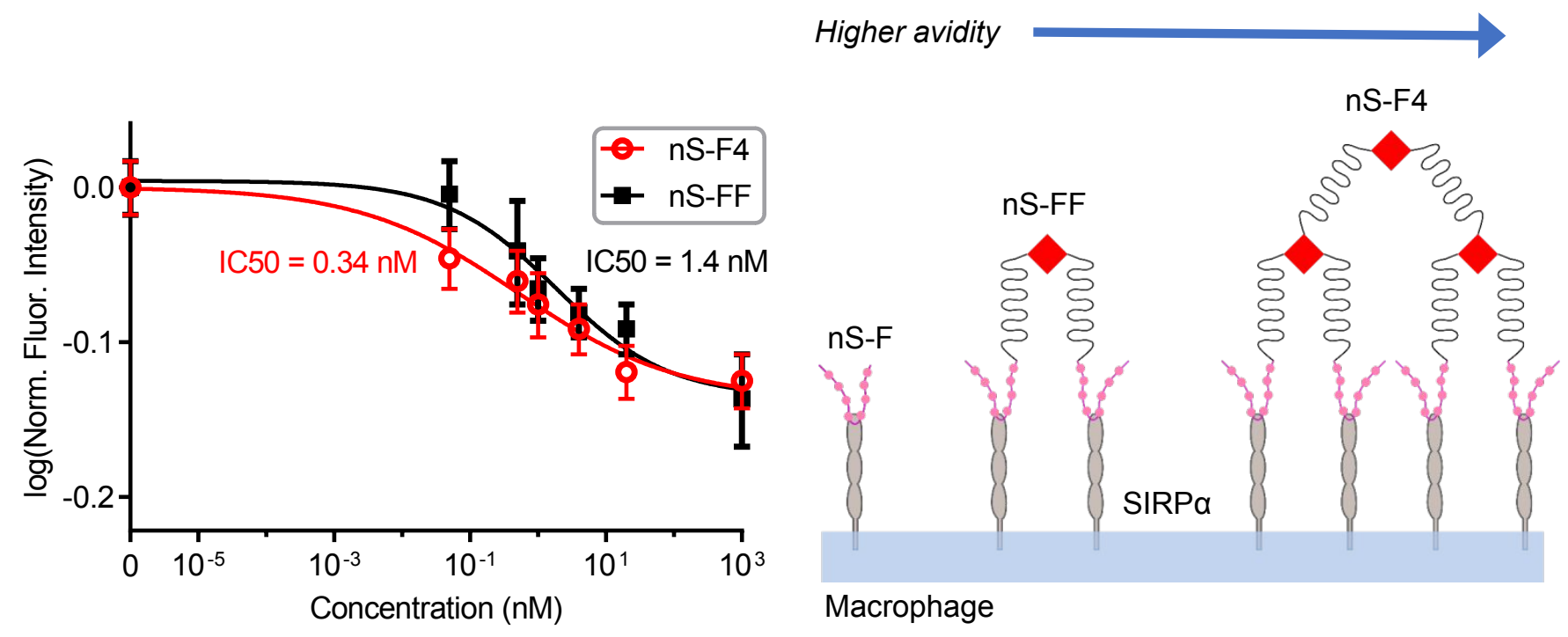

Figure S12: Avidity of SIRPa appears to scale with the increase of nano-Self multivalency

Tetravalent nS-F4 was more potent in inhibiting monovalent nS-F-fluor association with macrophages than bivalent nS-FF suggesting multivalency increases avidity of the nS-peptides to SIRPa. 


\section{References}

1. Tsai, R. K.; Discher, D. E. Inhibition of "Self" Engulfment through Deactivation of Myosin-II at the Phagocytic Synapse between Human Cells. J. Cell Biol. 2008, 180, 989-1003.

2. Hayes, B. H.; Tsai, R. K.; Dooling, L. J.; Kadu, S.; Lee, J. Y.; Pantano, D.; Rodriguez, P. L.; Subramanian, S.; Shin, J.; Discher, D. E. Macrophages Eat More after Disruption of Cis Interactions between CD47 and the Checkpoint Receptor SIRPa. J. Cell. Sci. 2020, jcs.237800. 